\title{
Lokalize Prostat Kanserinde Cerrahi Tedavinin Geleceği
}

\author{
Ömer Aytaç, H. Hüseyin Tavukçu, Fatih Atuğ
}

İstanbul Bilim Üniversitesi, Tıp Fakültesi, Üroloji Anabilim Dalı, İstanbul

\section{Giriş}

P rostat kanseri Amerika Birleşik Devletlerinde en fazla tanı konulan kanser türüdür. Akciğer kanseri ardından kansere bağlı ölümlerde ise ikinci sırada yer almaktadır $(1,2)$. İlk kez Walsh ve Donker tarafından tarif edilen; Açık Radikal Prostatektomi (ARP), prostat kanseri tedavisinde çı̆̆ır açmıştır $(3,4)$. Ancak $\mathrm{ARP}^{\prime}$ de insizyonun büyüklüğü, ağrının şiddeti, kanama miktarının fazlalığı, uzun hastane kalış ve kateterizasyon süresi, radikal prostatektomide yeni tedavi yöntemlerinin arayışını da beraberinde getirmiştir.

Açık radikal prostatektomi de yaşanan bu sıkıntıların giderilmesini amaçlayan minimal invaziv cerrahi yaklaşımlar olan laparoskopik (LRP) ve robotik radikal prostatektominin (RRP) geliştirilmesi ile birlikte daha az morbidite ve fonksiyonel sonuçlar elde edilmiştir.

İlk kez 1991 yılında Schuessler ve ark. (5) tarafından gerçekleştirilen laparoskopik cerrahinin, iki boyutlu görüntü sağlaması, dik ve uzun bir öğrenim eğrisinin bulunması gibi sıkıntıları mevcuttur. Günümüzde birçok merkezde gerçekleştirilen LRP, kompleks bir cerrahi teknik olarak kabul edilmektedir. Bu problemlerin aşılabilmesi amacıyla robot yardımlı laparoskopik cerrahi geliştirilmiştir.

İlk kez Abbou ve ark. (6) tarafından gerçekleştirilen robotik cerrahi laparoskopik cerrahiye göre önemli avantajlar sağlamaktadır. Bu avantajlar; anatomik yapıların 3 boyutlu ve 10 kata kadar büyütülerek görüntü sağlayabilmesi, robotik kolların 7 dereceye kadar yüksek hareket kabiliyeti sunması ve laparoskopik cerrahiye göre daha ergonomik yapıda olmasıdır. Bu avantajlar sayesinde robotik cerrahi LRP'ye göre daha kısa öğrenim eğrisi sağlamıştır. ABD'de 2011 yılı içinde tüm radikal prostektomi operasyonlarının \%80'inden daha fazlasının robotik cerrahi ile gerçekleştirilmiş olması ürologların da bu yeni teknolojiye hızlı bir şekilde adapte olduklarını göstermektedir (7).

Yapılan çalışmalarda robotik cerrahide ARP' ye oranla daha az kanama miktarı, daha az ağrı, daha kısa hastanede kalış ve kateterizasyon süresi rapor edilmiştir $(8,9)$. Ayrıca LRP ile karşılaştırıldığında daha iyi erektil fonksiyon ve idrar kontinans sonuçları bildirilmektedir $(10,11)$.

\section{RRP Operasyonu ve Cerrahi Tekniklerin Gelişimi}

Robotik cerrahinin sağladığı teknik avantajlar ile prostat anatomisinin daha iyi anlaşılması sayesinde, onkolojik sonuçların yanısıra hastaların yaşam kalitesini artıran idrar kontinansı ve erektil fonksiyona yönelik yeni cerrahi teknikler tanımlanmıştır

\section{İdrar Kontinansını Artıran Cerrahi Teknikler}

Radikal prostatektomide robotik cerrahinin gelişmesi ile birlikte idrar kontinansının erken dönemde ve tamamen kazanılmasını amaçlayan yeni cerrahi teknikler bildirilmiştir. Tablo 1'de RRP de idrar kontinansının korunmasına yönelik tanımlanan cerrahi teknikler ve sonuçları özetlenmiştir.

\section{Arka Rekonstrüksiyon Tekniği}

Robotik Radikal Prostatektomide vezikoüretral anastomoz öncesi rabdosfinkterin posterior katının Denonvillier fasyası ve mesane arka yüzüne dikilmesi olarak tarif edilen arka rekonstrüksiyon cerrahi tekniği, vezikoüretral anastamoz gerginliğini azaltmakta ve mesane boynuna destek sağlamaktadır. Rocco ve ark. RRP'de arka rekonstrüksiyon

Tablo 1: RRP de idrar kontinansının korunmasına yönelik tanımlanan cerrahi teknikler ve sonuçları

\begin{tabular}{|c|c|c|}
\hline Cerrahi Teknik & Yil & Sonuçlar \\
\hline Arka rekonstrüksiyon & 2008 & $\begin{array}{l}\text { Kontinansa kadar geçen sürenin kısaltılmasını ve anastamoz kaçaklarının } \\
\text { azaltılmasını sağlamaktadır }(12,13) \text {. }\end{array}$ \\
\hline Ön rekonstrüksiyon & 2009 & $\begin{array}{l}\text { Kaçırma basıncının değerini arttırır ve kontinansın hızlı geri kazanılmasında rol oynar (14). } \\
\text { 3. ayda kontinansın kazanılmasında etkilidir (15). }\end{array}$ \\
\hline $\begin{array}{l}\text { Ön ve arka (Total) } \\
\text { rekonstrüksiyon }\end{array}$ & 2009 & $\begin{array}{l}\text { Kontinansın daha erken dönemde ve yüksek oranda kazanılmasını sağladığı bildirilmektedir } \\
(16-18) \text {. Uzun dönem fark gösterilememiştir. }\end{array}$ \\
\hline $\begin{array}{l}360^{\circ} \text { Mesane boynu } \\
\text { koruyucu }\end{array}$ & 2013 & Kontinansın daha erken dönemde ve daha yüksek oranda kazanılmasını sağlamaktadır (19-21). \\
\hline Termal soğutma & 2009 & $\begin{array}{l}\text { Operasyon süresince pelvik bölgenin soğutulması erken dönem tam kontinans oranlarını } \\
\text { önemli ölçüde arttırmaktadır (22). }\end{array}$ \\
\hline
\end{tabular}


uyguladıkları hasta grubunda daha erken dönemde idrar kontinansı sağladıklarını bildirmişlerdir (12).

\section{Ön ve Arka (Total) Rekonstrüksiyon Tekniği}

Ön ve arka rekonstrüksiyonun birlikte uygulanması total rekonstrüksiyon olarak adlandırılmaktadır. Atuğ ve ark. yaptıkları bir çalışmada total rekonstrüksiyon uygulanmasının erken dönem idrar kontinans oranlarını arttırdığını, ancak uzun dönem sonuçlar arasında farklılık gözlenmediğini bildirmişlerdir (18).

\section{$360^{\circ}$ Mesane Boynu Koruyucu Teknik}

İdrar kontinansını iyileştirmeye yönelik tanımlanan tekniklerden biri olan mesane boynu koruyucu cerrahi teknik; mesane ve prostat sinırı belirlenerek bu seviyede yapilan ince diseksiyonlar ile mesane boynunun ortaya konulması olarak tarif edilmiştir. Mesane boynunun anatomik yapısının korunması sayesinde mesane boynu rekonstrüksiyonunun gerekmemesi ve internal sfinkterik mekanizmanın korunması sonucu erken dönemde ve yüksek oranda kontinans sağlandığ bildirilmiştir (19-21). (Şekil 1: RRP de 360 mesane boynu koruyucu cerrahi teknik ameliyat görüntüsü (20))

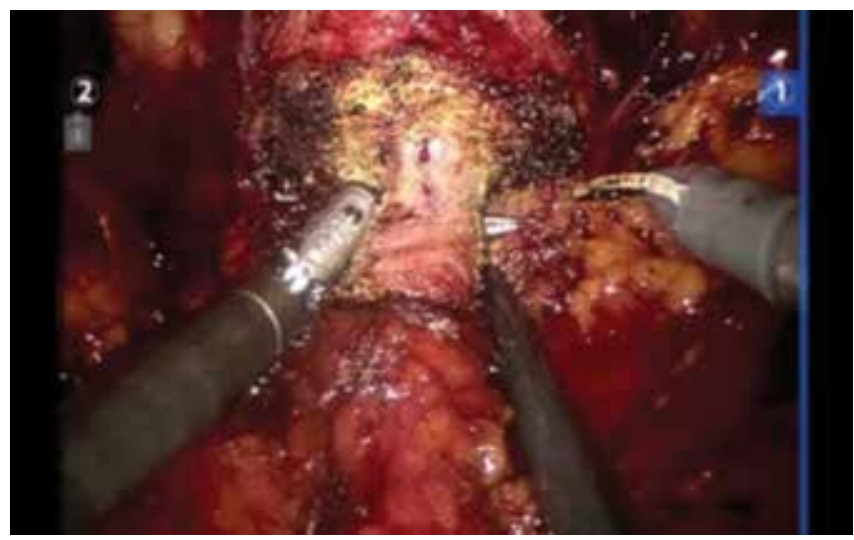

Şekil 1: RRP'de $360^{\circ}$ Mesane boynu koruyucu cerrahi teknik (20)

\section{Termal Soğutma Tekniği}

Radikal prostatektomi sonrası idrar kontinansını iyileştirici diğer bir teknik olan termal soğutma tekniği Finley ve ark. tarafından tanımlanmıştır. RRP'de endorektal soğutucu balon ile pelvik bölgede hipotermi oluşturulmasının ( $<30 \mathrm{Co})$ mesane, üretra ve nörovasküler demette (NVD) gelişen inflamatuar hasarı azalttığını ve bu sayede tam idrar kontinansının daha erken dönemde sağlandığını rapor etmişlerdir (22).

\section{Erektil Fonksiyonu İyileştirici Cerrahi Teknikler}

Robotik Radikal prostatektomide erektil fonksiyonun korunmasına amaçlayan cerrahi teknikler Tablo 2'de özetlenmiştir.

\section{Veil of Aphrodite Tekniği}

Radikal prostatektomide Menon ve ark. tarafından ilk olarak 2002 yılında tarif edilen ve NVD'in korunmasını amaçlayan "Veil of Aphrodite" diğer ismiyle intrafasyal teknik ile erektil fonksiyon sonuçlarında önemli oranda iyileşmeler sağlanmıştır (23).

Nörovasküler demet koruyucu radikal prostatektomide "Veil of Aphrodite" olarak adlandırılan intrafasyal teknik; prostat kapsülü ile periprostatik fasya arasından yapılan diseksiyon, interfasyal teknik ise; periprostatik fasya ile levator ani viseral fasyası arasından yapılan diseksiyon olarak tarif edilmektedir. Yapılan çalışmalarla NVD'nin intrafasyal teknik ile büyük oranda korunduğu bildirilmiştir (27).

\section{Atermal Diseksiyon Tekniği}

Erektil fonksiyonun korunmasında NVD diseksiyonunun atermal olarak yapılmasının etkisi bilinmektedir. Ahlering ve ark. radikal prostatektomi de hipogastrik artere bulldog klemp yerleştirilerek koter kullanımının en az düzeye indirilmesinin erektil fonksiyon iyileşmesini 5 kat artırdığını rapor etmişlerdir (25).

\section{NVD Geriliminin Önlenmesi}

NVD'nin operasyon esnasında yaralanması ya da aşırı traksiyonu sonucu nöropraksi gelişmesi erektil fonksiyon sonuçlarını olumsuz yönde etkilemektedir. Kowalczyk ve ark. traksiyona bağlı nöropraksi etkisini araştırdıkları çalışmalarında, traksiyon yapılmayan hastalarda erektil fonksiyonun daha erken dönemde düzeldiğini bildirmişlerdir. 5 . ayda yapılan kontrollerde potans oranları sirası ile traksiyon uygulananlarda \%28 iken traksiyon uygulanmayanlarda \%48 olarak bildirilmiştir. Bu hastaların 1. yıl takip oranları değerlendirildiğinde ise her iki grup arasında farklılık olmadığ rapor edilmiştir (26).

\section{Yeni Nesil Robotik Sistemler ve Yöntemler}

Cerrahi operasyonlarda robot teknolojisi ilk kez 1988'de Kwoh ve ark. tarafından stereotaktik beyin cerrahi operasyonunda robotik kolun kullanılmasıyla başlamıştır. Robotik teknolojinin üroloji de ilk kullanımı, modifiye edilen bu robotik kolun prostatın transüretral rezeksiyonu operasyonunda kullanılması olarak kabul edilmektedir (28).

Tablo 2: RRP de erektil fonksiyonun korunmasına yönelik tanımlanan cerrahi teknikler ve sonuçları

\begin{tabular}{lll}
\hline Cerrahi Teknik & Yıl & Sonuçlar \\
\hline Veil of Aphrodite & 2002 & Erektil fonksiyonun erken dönemde kazanılmasını sağlamaktadır (23). \\
Atermal diseksiyon & 2007 & $\begin{array}{l}\text { NVD korunması diğer sonuçları etkilemeksizin temel erektil fonksiyonun geri kazanılmasını } \\
\text { sağlamaktadır (24). } \\
\text { NVD diseksiyonunda termal hasarlanmadan kaçınılması erektil fonksiyonun erken dönemde } \\
\text { düzelmesinde etkilidir (25). }\end{array}$
\end{tabular}
NVD’nin traksiyonunundan kaçınılması erektil fonksiyonun geri kazanılmasında önemlidir (26). 
Günümüze kadar geliştirilen robotik sistemler çalışma prensiplerine göre aktif (29), semi-aktif (28) ve master-slave sistemler $(30,31)$ olarak sinıflandırılmaktadır. Aktif sistemler cerrahın kontrolü altında bir işlemi otonom olarak yerine getirirken, semi-aktif sistemler otonom yapıları yanında cerrah tarafından da yönetilme özelliğine sahiptirler.

En gelişmiş robotik sistem olan master-slave sistemlerin otonom özelliği bulunmamaktadır. Bu sistemin çalışma prensibi; robot kolların bulunduğu robotik ünite ve cerrahın el hareketlerini birebir taklit edilmesini sağlayan konsoldan oluşmaktadır. Günümüze kadar geliştirilmiş olan master-slave sistemler; Aesop, Zeus ve da Vinci olmakla birlikte bunlar içinde en sık kullanılan ve en fazla kabul görmüş olan robotik sistem da Vinci'dir.

Günümüzde robotik cerrahide en yeni gelişme olan ve gelecek dönemlerde klasik çok portlu giriş yerine geçeceği düşünülen Laparo-endoskopik tek port tedavi yöntemi ilk kez Hirano ve ark. tarafından retroperitoneal adrenalektomi operasyonunda standart laparoskopik aletler ile gerçekleştirilmiştir (32).

Tek insizyondan laparoskopik radikal prostatektomi ameliyatını fleksibl laparoskopik enstrumanlar kullanarak ilk defa gerçekleştiren Kaouk ve ark. radikal prostatektomi de tek insizyon tekniğinin uygulanabilirliğini göstermişlerdir (33). Tekniğin ergonomik olmayan yapısına bağlı yaşanan aletlerde çarpışma, sütür atmada ve dokuların ayrıştırılmasındaki güçlüklere dikkat çekerek, daha uygun port ve cerrahi aletlerin geliştirilmesi gerekliliğini bildirmişlerdir. Ayrıca bu yöntemin robotik olarak gerçekleştirilmesinin daha uygun olduğunu ifade etmişlerdir.

Robotik cerrahi gerçek anlamda yerini üroloji ameliyatları içinde en sık uygulandığ radikal prostatektomide bulmuştur.

Robotik Radikal Prostatektominin ilk defa tek porttan uygulanması, 2009 yılında Kaouk ve ark. tarafından bildirilmiştir. Artikülasyon özelliği olan enstrumanlar kullanılarak gerçekleştirdikleri ameliyatlarında, robot kullanımının tek port kullanımındaki mevcut sıkıntıları azalttığını bildirmişlerdir (34).

Robotik cerrahideki son güncel gelişmeler 1şığında Intuitive firması, özelikle ürolojik ameliyatlar için geliştirdiğini bildirdiği da Vinci SP (single port) robotik sistemini 2015'in 2. yarısında kullanıma sunulacağını ilan etmiştir (35). (Şekil 2) Ameliyatların 25 mm'lik tek port içinden gerçekleştirilecek şekilde planlandığını ve bu nedenle erişkin hastalarda kullanılabileceği bildirilmektedir.

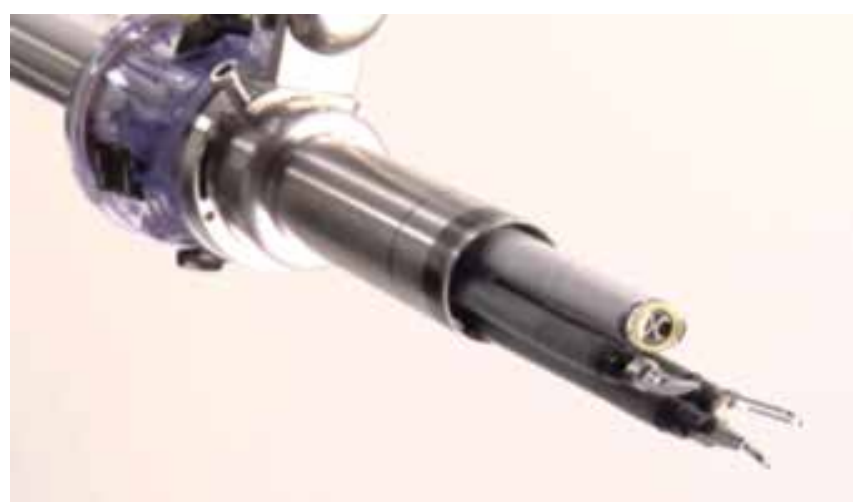

Şekil 2: RRP'de $360^{\circ}$ Mesane boynu koruyucu cerrahi teknik (20)
Günümüzde hızla gelişen tek port cerrahi yönteminde robotik sistemin kullanılması ile teknik açıdan birçok kolaylık sağlanması yanında hastalar için de daha az ağrı, daha hızlı iyileşme ve daha iyi kozmetik sonuçlar sunacağı öngörülmektedir $(36,37)$.

İntuitive firmasının robotik cerrahi sistemde tek konumda olmasını sağlayan patentlerin yasal süresinin 2016 yılında dolacağı ve halihazırda intutive firması tarafından geliştirilen bu teknolojilerin rakip firmalar tarafından da kullanılabileceği bilinmektedir. Da vinci robotik sistemin yaygın kullanımına bağlı teknik bilgilerin ayrıntılı öğrenilmesi, rakip firmaların benzer ya da daha gelişmiş ürünleri kullanıma sunmasının, mevcut dengelerin değişimine neden olabileceği düşünülmektedir (38).

\section{Kaynaklar}

1. Meng MV, Elkin EP, Harlan SR, Mehta SS, Lubeck DP, Carroll PR. Predictors of treatment after initial surveillance in men with prostate cancer: results from CaPSURE. The Journal of urology. 2003;170(6 Pt 1):227983.

2. Altekruse S, Kosary C, Krapcho M, Neyman N, Aminou $\mathrm{R}$, Waldron W, et al. SEER cancer statistics review, 19752007. Bethesda, MD: National Cancer Institute. 2010;7.

3. Walsh P. Impotence following radical prostatectomy: insight into etiology and prevention. J urol. 1982;128:492-7.

4. Brandeis J, Pashos CL, Henning JM, Litwin MS. A nationwide charge comparison of the principal treatments for early stage prostate carcinoma. Cancer. 2000;89(8):1792-9. PubMed PMID: 11042575.

5. Schuessler W, Kavoussi L, Clayman R, Vancaille T. Laparoscopic radical prostatectomy: initial case report. The Journal of urology. 1992;147(4):246.

6. Abbou CC, Hoznek A, Salomon L, Olsson LE, Lobontiu A, Saint F, et al. Laparoscopic radical prostatectomy with a remote controlled robot. The Journal of urology. 2001;165(6 Pt 1):1964-6. PubMed PMID: 11371890.

7. Piechaud P. State of the art: urologic surgery. Journal of visceral surgery. 2011;148(5 Suppl):e27-9. doi: 10.1016/j. jviscsurg.2011.08.004. PubMed PMID: 22056796.

8. Patel VR, Palmer KJ, Coughlin G, Samavedi S. Robotassisted laparoscopic radical prostatectomy: perioperative outcomes of 1500 cases. Journal of endourology / Endourological Society. 2008;22(10):2299-305. doi: 10.1089/end.2008.9711. PubMed PMID: 18837657.

9. Ficarra V, Novara G, Artibani W, Cestari A, Galfano A, Graefen M, et al. Retropubic, laparoscopic, and robot-assisted radical prostatectomy: a systematic review and cumulative analysis of comparative studies. European urology. 2009;55(5):1037-63. doi: 10.1016/j. eururo.2009.01.036. PubMed PMID: 19185977.

10. Sandoval Salinas C, González Rangel AL, Cataño Cataño JG, Fuentes Pachón JC, Castillo Londoño JS. Efficacy of Robotic-Assisted Prostatectomy in Localized Prostate Cancer: A Systematic Review of Clinical Trials. Advances in urology. 2013;2013.

11. Coelho RF, Rocco B, Patel MB, Orvieto MA, Chauhan $\mathrm{S}$, Ficarra V, et al. Retropubic, laparoscopic, and robotassisted radical prostatectomy: a critical review of outcomes reported by high-volume centers. Journal of Endourology. 2010;24(12):2003-15. 
12. Rocco F, Carmignani L, Acquati P, Gadda F, Dell'Orto P, Rocco B, et al. Restoration of posterior aspect of rhabdosphincter shortens continence time after radical retropubic prostatectomy. The Journal of urology. 2006;175(6):2201-6. doi: 10.1016/S0022-5347(06)00262-X. PubMed PMID: 16697841.

13. Coelho RF, Chauhan S, Orvieto MA, Sivaraman A, Palmer KJ, Coughlin G, et al. Influence of modified posterior reconstruction of the rhabdosphincter on early recovery of continence and anastomotic leakage rates after robot-assisted radical prostatectomy. European urology. 2011;59(1):72-80. doi: 10.1016/j.eururo.2010.08.025. PubMed PMID: 20801579.

14. Campenni MA, Harmon JD, Ginsberg PC, Harkaway RC. Improved continence after radical retropubic prostatectomy using two pubo-urethral suspension stitches. Urologia internationalis. 2002;68(2):109-12.

15. Patel VR, Coelho RF, Palmer KJ, Rocco B. Periurethral suspension stitch during robot-assisted laparoscopic radical prostatectomy: description of the technique and continence outcomes. European urology. 2009;56(3):472-8.

16. Hurtes X, Rouprêt M, Vaessen C, Pereira H, Faivre d'Arcier B, Cormier L, et al. Anterior suspension combined with posterior reconstruction during robotassisted laparoscopic prostatectomy improves early return of urinary continence: a prospective randomized multicentre trial. BJU international. 2012;110(6):875-83.

17. Koliakos N, Mottrie A, Buffi N, De Naeyer G, Willemsen $\mathrm{P}$, Fonteyne E. Posterior and anterior fixation of the urethra during robotic prostatectomy improves early continence rates. Scandinavian journal of urology and nephrology. 2010;44(1):5-10.

18. Atug F, Kural AR, Tufek I, Srivastav S, Akpinar H. Anterior and posterior reconstruction technique and its impact on early return of continence after robotassisted radical prostatectomy. Journal of Endourology. 2012;26(4):381-6.

19. Nyarangi-Dix JN, Radtke JP, Hadaschik B, Pahernik $S$, Hohenfellner $M$. Impact of complete bladder neck preservation on urinary continence, quality of life and surgical margins after radical prostatectomy: A randomized, controlled, single blind trial. The Journal of urology. 2013;189(3):891-8.

20. Fatih Atuğ HHT, Ömer Aytaç. Robotik Radikal Prostatektomide 360 derece Mesane Boynu Koruyucu Cerrahi Teknik. video sunum VS-102014.

21. H.Hüseyin Tavukçu ÖA, Fatih Atuğ. Robot Yardımlı Laparoskopik Radikal Prostatektomide Tam Kontinans İçin Neler Yapılmalı? 32014.

22. Finley DS, Osann K, Chang A, Santos R, Skarecky D, Ahlering TE. Hypothermic robotic radical prostatectomy: impact on continence. Journal of Endourology. 2009;23(9):1443-50.

23. Menon M, Tewari A, Peabody J. Vattikuti Institute prostatectomy: technique. The Journal of urology. 2003;169(6):2289-92.

24. Tewari AK, Srivastava A, Huang MW, Robinson BD, ShevchukMM, Durand M, etal. Anatomical grades of nerve sparing: a risk-stratified approach to neural-hammock sparing during robot-assisted radical prostatectomy (RARP). BJU international. 2011;108(6b):984-92.
25. Ahlering TE, Rodriguez E, Skarecky DW. Overcoming obstacles: nerve-sparing issues in radical prostatectomy. Journal of Endourology. 2008;22(4):745-50.

26. Kowalczyk KJ, Huang AC, Hevelone ND, Lipsitz SR, $\mathrm{Yu} \mathrm{H}-\mathrm{y}, \mathrm{Ulmer} W \mathrm{~W}$, et al. Stepwise approach for nerve sparing without countertraction during robot-assisted radical prostatectomy: technique and outcomes. European urology. 2011;60(3):536-47.

27. Menon M, Shrivastava A, Kaul S, Badani KK, Fumo M, Bhandari M, et al. Vattikuti Institute prostatectomy: contemporary technique and analysis of results. European urology. 2007;51(3):648-58.

28. Davies B, Hibberd R, Ng W, Timoney A, Wickham J. The development of a surgeon robot for prostatectomies. Proceedings of the Institution of Mechanical Engineers, Part H: Journal of Engineering in Medicine. 1991;205(1):358.

29. Paul HA, Bargar WL, Mittlestadt B, Musits B, Taylor RH, Kazanzides P, et al. Development of a surgical robot for cementless total hip arthroplasty. Clinical Orthopaedics and related research. 1992;285:57-66.

30. Su L-M, Stoianovici D, Jarrett TW, Patriciu A, Roberts WW, Cadeddu JA, et al. Robotic percutaneous access to the kidney: comparison with standard manual access. Journal of Endourology. 2002;16(7):471-5.

31. Kavoussi LR, Moore RG, Partin AW, Bender JS, Zenilman ME, Satava RM. Telerobotic assisted laparoscopic surgery: initial laboratory and clinical experience. Urology. 1994;44(1):15-9. PubMed PMID: 8042260.

32. Hirano D, Minei S, Yamaguchi K, Yoshikawa T, Hachiya $\mathrm{T}$, Yoshida T, et al. Retroperitoneoscopic adrenalectomy for adrenal tumors via a single large port. Journal of endourology. 2005;19(7):788-92.

33. Kaouk JH, Goel RK, Haber G-P, Crouzet S, Desai MM, Gill IS. Single-port laparoscopic radical prostatectomy. Urology. 2008;72(6):1190-3.

34. Kaouk JH, Goel RK, Haber GP, Crouzet S, Stein RJ. Robotic single-port transumbilical surgery in humans: initial report. BJU international. 2009;103(3):366-9.

35. intuitive surgical i, sunnyvalley, CA. Available from: http://investor.intuitivesurgical.com/phoenix. zhtml?c=122359\&p=irol-newsArticle\&ID=1920546.

36. Box G, Averch T, Cadeddu J, Cherullo E, Clayman R, Desai $\mathrm{M}$, et al. Nomenclature of natural orifice translumenal endoscopic surgery (NOTES ${ }^{\mathrm{TM}}$ ) and laparoendoscopic single-site surgery (LESS) procedures in urology. Journal of Endourology. 2008;22(11):2575-82.

37. Gettman MT, White WM, Aron M, Autorino R, Averch T, Box G, et al. Where do we really stand with LESS and NOTES? European urology. 2011;59(2):231-4.

38. Hoffman A. Intuitive Surgical, Inc.: How Long Can Their Monopoly Last? 2010.

Yazışma Adresi:

Ömer Aytaç

İstanbul Bilim Üniversitesi, Tıp Fakültesi,

Üroloji Anabilim Dalı, İstanbul

Tel: +905065336097

e-mail:droaytac@gmail.com 